\title{
Some demonstrations of the complementary functioning of the eyes
}

\author{
W. J. M. LEVELT ${ }^{1}$ \\ INSTITUTE FOR PERCEPTION RVO-TNO, SOESTERBERG, THE NETHERLANDS
}

The eyes have complementary shares in the production of binocular brightness. Artificial increase of the contribution of one eye automatically leads to an equal decrease of the contribution of the second eye. The responsible mechanism for increase and decrease of shares is called 'contour mechanism.' Its functioning is explained by means of two stereoscopic patterns.

If the two eyes are presented with identical fields of equal luminance $\left(\mathrm{E}_{\mathrm{b}}\right)$, and one increases the luminance of the left field (up to $\mathbf{E}_{1}$ ), one may keep the apparent binocular brightness constant by simultaneously decreasing the luminance of the right field to some degree (to $E_{r}$, say). In fact, binocular brightness appears to be constant as long as a sum of weighted monocular luminances is kept constant; in formula: $w_{1} E_{1}+w_{r} E_{r}=C$ (see Levelt, 1965b). The brightness impression is the same as if both fields were of luminance $\mathbf{E}_{\mathrm{b}} \cdot \mathrm{w}_{1}$ and $\mathrm{w}_{\mathrm{r}}$ are weighting coefficients for the left and the right eye respectively. They are dependent on eye dominance, but can also be varied by artificial means. An artificial increase in the contribution of an eye to the binocular brightness impression can be produced by putting some contour (for instance a circle) in the field of that eye: the weighting coefficient is increased for that part of the monocular field, then.

It appears that, if $w_{1}$ is increased in this way (a contour in the field of the left eye only), $w_{r}$ decreases to the same amount (Levelt 1965a, b). This has been called the law of complementary shares. If the sum of the contributions of the eyes is fixed at unity, the law of complementary shares can be written as: $w_{1}+w_{r}=1$, independent of the ratio of $w_{1}$ and $w_{r}$.

It is furthermore possible to show, that the smaller the distance $d$ between the fixation point of an eye and a monocular contour in the field of this eye, the more the weighting coefficient of this eye tends to unity in the point of fixation. This may be called the contour mechanism: $w \rightarrow 1$, if $d \rightarrow 0$ for an eye (and thus $w \rightarrow 0$ for the other eye). This means that, in the immediate environment of a monocularly presented contour $\left(-0.5^{\circ}\right)$, the binocular brightness is exclusively determined by the luminance in this monocular field, and is independent of the luminance of the contralateral field (Levelt, 1965a, b).

It is the aim of this paper to show, that what has been measured by exact optical means, can also be demonstrated by simple stereoscopic pictures.

Binocular fusion of the patterns in Fig. 1 produces the following paradoxical situation. For the sake of
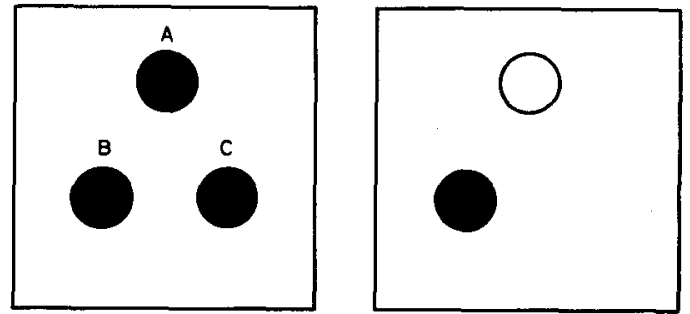

Fig. 1. A brightness paradox. Discs $B$ and $C$ are about equal in stereoscopic brightness, whereas $A$ appears to be much brighter than $\mathrm{C}$.

simplicity, the luminance of the black discs is supposed to be zero, whereas the bright field has luminance 1 . For disc A a contour is present in both monocular fields, therefore the weighting coefficients $w_{1}$ and $w_{r}$ are both equal to $1 / 2$ (disregarding eye dominance). The perceived binocular brightness of this disc is thus equal to the brightness of a normal binocular field of luminance $\mathbf{E}_{\mathbf{b}}=1 / 2.0+1 / 2.1=1 / 2$ ('grey'). Disc $C$, which has the same luminance as $A$ in the left and right field respectively, will nevertheless look differently. For, there is a contour in the left field only, and thus $w_{1} \rightarrow 1$ according to the contour mechanism. If $w_{1}=1$, this means that the brightness impression will be equivalent to that produced by a binocular field of luminance $E_{b}=1.0+0.1=0$, so for $C$ it should be the case that $\mathrm{E}_{\mathrm{b}} \rightarrow 0$ (nearly black). Although disc $\mathrm{B}$ is black in the right field, whereas $C$ is white in the right field, the appearance of $B$ and $C$ will not differ very much. The equivalent binocular luminance for $B$ is $\mathrm{E}_{\mathrm{b}}=1 / 2 \cdot 0+1 / 2 \cdot 0=0$ ('black'). These predictions can easily be confirmed by binocular fusion of the patterns. Note, however, that mainly the brightness of $A$ is subject to eye-dominance.

This type of pattern can be produced at will, according to the same principles. Another example is given in Fig. 2, which has been designed as an argument against the Gestalt view on binocular rivalry. According to this view, a Gestalt cannot be deformed by binocular interaction (see Gellhorn, 1924). It can only be present or absent in toto. However, the complementary shares theory predicts distortion of the black bar in Fig. 2 in stereoscopic vision. For, the left part is equivalent to disc $\mathrm{A}$ in Fig. 1 (producing a grey appearance), whereas the right part of the bar is equivalent to $C$ (which appeared to be black). The prediction is therefore, that the bar looks grey in the left half, shading into black in the right half: a clear distortion of the 


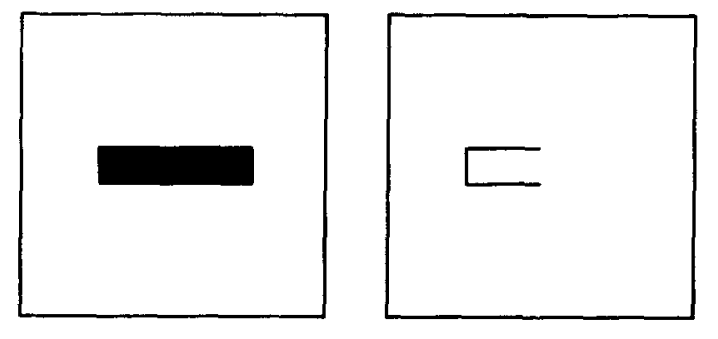

Fig. 1. An argument against the Gestalt view on rivalry. The Gestalt of the bar is disturbed, stereoscopically. The left half appears grey, shading into black in the right half.

Gestalt. That this prediction is borne out by stereoscopic inspection of the figure shows again that binocular interaction is functionally prior to Gestalt formation (see also Julesz, 1965).

The contour mechanism and the law of complementary shares necessarily come into conflict if non-corresponding contours are presented to the two eyes. For instance, in the situation of a binocular crossing of monocular contours, the contour mechanism causes both $w_{1} \rightarrow 1$ and $w_{r} \rightarrow 1$. In and near the crossing point this is incompatible with the law of complementary shares $\left(w_{1}+w_{r}=1\right)$. This is the perceptual conflict underlying binocular rivalry. The conflict is apparently solved by $w_{1}$ and $w_{r}$ being 1 in turn, so saving the law of complementary shares by an alternation process. Of course, there is no reason to assume a different mechanism for the normal fusion-situation, where the monocular patterns are congruent (see Levelt, 1965b).

\section{References}

Gellhom, E. Quantitative Untersuchungen über den Wettstreit der Sehfelder. III. Zur Kenntnis der psychologischen Ursachen des Wettstreites. Pflügers Archiv für die gesammte Physiologie, $1924,206,237-249$.

Julesz, B. Texture and visual perception. Scient. American, 1965. Feb., 38-48.

Levelt, W. J. M. Binocular brightness averaging and contour in formation. Brit. J. Psychol., 1965a, 56, 1-13.

Levelt, W. J. M. On binocular rivalry. Institute for Perception RVO-TNO edition, $1965 \mathrm{~b}$

\section{Hote}

1. Now at the Center for Cognitive Studies, Harvard University. 